\title{
Piezo actuators for Nanomedicine research
}

\begin{abstract}
The characteristics, the structural scheme and the matrix transfer function of the piezo actuator for the nanomedicine research are obtained. The transfer functions of the piezo actuator are described the characteristics of the actuator with regard to its physical parameters and external load.
\end{abstract}

Keywords: piezo actuator, structural scheme, transfer function
Volume 3 Issue 2 - 2019

\begin{abstract}
Afonin SM
National Research University of Electronic Technology (MIET), Russia

Correspondence: Afonin Sergey Mikhailovich, National Research University of Electronic Technology (MIET), I24498, Moscow, Russia, Email learner0I@mail.ru
\end{abstract}

Received: February 21, 2019 | Published: March 25, 2019

\section{Introduction}

The piezo actuator is the piezo mechanical device intended for actuation of the mechanisms, the systems or the management based on the piezo effect, converts electrical signals into mechanical movement or force. The piezo actuators for nano and micro displacement are provided the movement range from several nanometers to tens of microns, the sensitivity of up to $10 \mathrm{~nm} / \mathrm{V}$, the loading capacity of up to $1000 \mathrm{~N} .{ }^{1-5}$ The piezo actuator is used in the majority of the scanning tunneling microscopes and the atomic force microscopes. The mathematical model, the structural scheme and the transfer functions of the piezo actuator are calculated for designing the control mechatronics systems for the nanomedicine research. ${ }^{3-12}$ The structural scheme and transfer functions the piezo actuator make it possible to describe the dynamic and static properties of the piezo actuator for the nanomedicine research with regard to its physical parameters and external load. ${ }^{11-24}$

\section{Structural scheme}

The method of mathematical physics with Laplace transform is applied for the solution the wave equation. The structural scheme of the piezo actuator for the nanomedicine research is changed from Cady and Mason electrical equivalent schemes, ${ }^{6,7}$ of the piezo actuator. The equation of the inverse piezo effect, ${ }^{7,9,11}$ has form $S_{i}=d_{m i} \Psi_{m}+S_{i j}^{\Psi} T_{j}$,

where $S_{i}$ is the relative displacement along axis $i, \Psi_{m}=\left\{E_{m}, D_{m}\right\}$ is the control parameter, $E_{m}$ is the electric field strength for the voltage control along axis $m, D_{m}$ is the electric induction for the current control along axis $m, d_{m i}=\left\{d_{m i}, g_{m i}\right\}$ is the piezo module for the voltage control or for the current control, $T_{j}$ is the mechanical stress along axis $j, s_{i j}^{\Psi}$ is the elastic compliance for the control parameter $\Psi=$ const , the indexes $i=1,2, \ldots, 6 ; j=1,2, \ldots, 6 ; m=1,2,3$. The main size of the piezo actuator along axis $i$ is determined $l=\{\delta, h, b\}$ in form the thickness, the height, the width for the longitudinal, transverse, shift piezo effect. For the construction the structural scheme of the piezo actuator is used the wave equation for the wave propagation in the long line with damping but without distortions. With using Laplace transform is obtained the linear ordinary secondorder differential equation. The problem for the partial differential equation of hyperbolic type using the Laplace transform is reduced to the simpler problem for the linear ordinary differential equation, ${ }^{9,11,12}$

The structural scheme of the piezo actuator, ${ }^{4,9,11,12}$ on Figure 1 are determined, using method of the mathematical physics for the solution of the wave equation, the boundary conditions and the equation of the inverse piezo effect, where $S_{0}$ is the cross section area, $M_{1}$, $M_{2}$ are the mass of load, $\Xi_{1}(p), \Xi_{2}(p), F_{1}(p), F_{2}(p)$ are the
Laplace transforms of the displacements, the forces on the faces $1,2$. The structural schemes and the matrix transfer function of the voltage controlled or current controlled piezo actuator are obtained from its mathematical model. The deformations of the piezo actuator are described by the matrix transfer function.

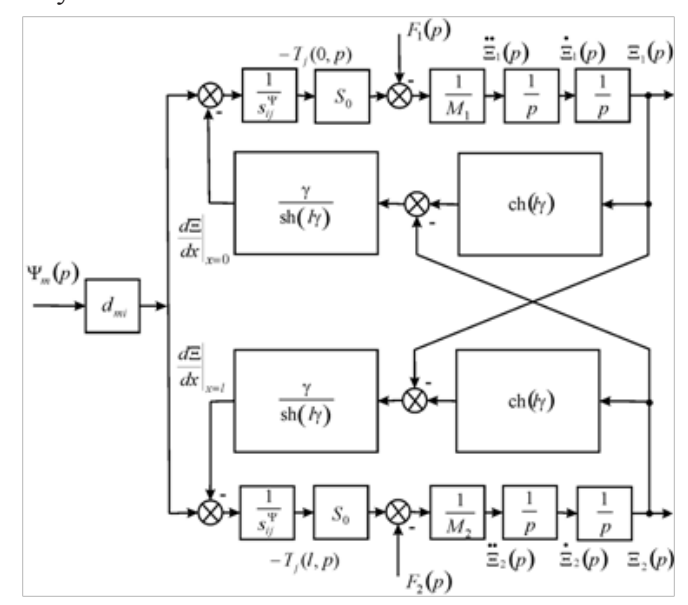

Figure I Structural scheme of piezo actuator for nanomedicine research.

\section{Conclusions}

The structural scheme, the transfer functions of the piezo actuators for the nanomedicine research are described the characteristics of the piezo actuator with regard to its physical parameters, external load.

\section{Acknowledgments}

None.

\section{Conflicts of interest}

Author declares no conflicts of interest.

\section{References}

1. Schultz J, Ueda J, Asada H. Cellular Actuators. Oxford: ButterworthHeinemann Publisher. 2017. p. 382.

2. Przybylski J. Static and dynamic analysis of a flextensional transducer with an axial piezoelectric actuation. Engineering Structures. 2015;84:140-151.

3. Afonin SM. Block diagrams of a multilayer piezoelectric motor for nanoand microdisplacements based on the transverse piezoeffect. Journal of Computer and Systems Sciences International. 2015;54(3):424-439.

4. Afonin SM. Structural parametric model of a piezoelectric nanodisplacement transduser. Doklady Physics. 2008;53(3):137-143. 
5. Afonin SM. Solution of the wave equation for the control of an elecromagnetoelastic transduser. Doklady Mathematics. 2006;73(2):307-313.

6. Cady WG. Piezoelectricity: An Introduction to the Theory and Applications of Eelectromechancial Phenomena in Crystals. New York, London: McGraw-Hill Book Company; 1946. p. 806.

7. Mason W. Physical Acoustics: Principles and Methods. Vol.1. Part A Methods and Devices, New York: Academic Press; 1964. p. 515.

8. Zwillinger D. Handbook of Differential Equations. Boston: Academic Press; 1989. p. 673.

9. Afonin SM. A generalized structural-parametric model of an elecromagnetoelastic converter for nano- and micrometric movement control systems: III. Transformation parametric structural circuits of an elecromagnetoelastic converter for nano- and micrometric movement control systems. Journal of Computer and Systems Sciences International. 2006;45(2):317-325

10. Afonin SM. Decision wave equation and block diagram of electromagnetoelastic actuator nano- and microdisplacement for communications systems. International Journal of Information and Communication Sciences. 2016;1(2):22-29.

11. Afonin SM. Structural-parametric model and transfer functions of electroelastic actuator for nano- and microdisplacement. Chapter 9 in Piezoelectrics and Nanomaterials: Fundamentals, Developments and Applications. Parinov IA, editor, New York: Nova Science; 2015. p. $225-242$.

12. Afonin SM. A structural-parametric model of electroelastic actuator for nano- and microdisplacement of mechatronic system. Chapter 8 in Advances in Nanotechnology. Volume 19. Bartul Z, Trenor J, editors, New York: Nova Science; 2017. p. 259-284.

13. Afonin SM. Electromagnetoelastic nano- and microactuators for mechatronic systems. Russian Engineering Research. 2018;38(12):938-944.

14. Afonin SM. Nano- and micro-scale piezomotors. Russian Engineering Research. 2012;32(7-8):519-522.
15. Afonin SM. Stability of strain control systems of nano-and microdisplacement piezotransducers. Mechanics of Solids. 2014;49(2):196-207.

16. Afonin SM. Structural-parametric model electromagnetoelastic actuator nanodisplacement for mechatronics. International journal of Physics. 2017;5(1):9-15.

17. Afonin SM. Structural-parametric model of piezoactuator nano- and microdisplacement for nanoscience. AASCIT Journal of Nanoscience. 2017;3(3):12-18.

18. Afonin SM. Solution wave equation and parametric structural schematic diagrams of electromagnetoelastic actuators nano- and microdisplacement. International Journal of Mathematical Analysis and Applications. 2016;3(4):31-38.

19. Afonin SM. Structural-parametric model of electromagnetoelastic actuator for nanomechanics. Actuators. 2018;7(1):1-9.

20. Afonin SM. Structural-parametric models and transfer functions of electromagnetoelastic actuators nano- and microdisplacement for mechatronic systems. International Journal of Theoretical and Applied Mathematics. 2016;2(2):52-59.

21. Afonin SM. Structural-parametric model of electro elastic actuator for nanotechnology and biotechnology. Journal of Pharmacy and Pharmaceutics. 2018;5(1):8-12.

22. Afonin SM. Structural-parametric model electroelastic actuator nanoand microdisplacement of mechatronics systems for nanotechnology and ecology research. MOJ Ecology \& Environmental Sciences. 2018;3(5):306-309.

23. Nalwa HS. Encyclopedia of Nanoscience and Nanotechnology. Los Angeles: American Scientific Publishers; 2004.

24. Bhushan B. Springer Handbook of Nanotechnology. New York: Springer; 2004. p. 1222. 